\title{
Next-to-next-to-leading order contributions to inclusive jet production in deep-inelastic scattering and determination of $\alpha_{s}$
}

\author{
Thomas Biekötter, ${ }^{1}$ Michael Klasen, ${ }^{1, *}$ and Gustav Kramer ${ }^{2}$ \\ ${ }^{1}$ Institut für Theoretische Physik, Westfälische Wilhelms-Universität Münster, \\ Wilhelm-Klemm-Straße 9, D-48149 Münster, Germany \\ ${ }^{2}$ II. Institut für Theoretische Physik, Universität Hamburg, \\ Luruper Chaussee 149, D-22761 Hamburg, Germany \\ (Received 31 August 2015; published 26 October 2015)
}

\begin{abstract}
We present the first calculation of inclusive jet production in deep-inelastic scattering with approximate next-to-next-to-leading order (aNNLO) contributions, obtained from a unified threshold resummation formalism. The leading coefficients are computed analytically. We show that the aNNLO contributions reduce the theoretical prediction for jet production in deep-inelastic scattering, improve the description of the final HERA data in particular at high photon virtuality $Q^{2}$ and increase the central fit value of the strong coupling constant.
\end{abstract}

DOI: 10.1103/PhysRevD.92.074037

PACS numbers: 12.38.Bx, 13.60.-r

\section{INTRODUCTION}

The HERA collider, which operated at DESY from 1992 to 2007, has produced many important physics results, including perhaps the most precise determinations to date of the quark and gluon densities in the proton from single experiments (H1, ZEUS) [1,2] and their combined data sets [3]. These data, taken in deep-inelastic electron-proton scattering, are complemented by a wealth of data from photoproduction at low virtuality $Q^{2}$ of the exchanged photon, in particular on jet production [4], giving access also to the distributions of partons in the photon [5] and to measurements of the strong coupling constant [6].

Using the data set of the HERA-II phase of the HERA collider from 2003-2007 with an integrated luminosity of $351 \mathrm{pb}^{-1}$, the $\mathrm{H} 1$ Collaboration have recently published final measurements of inclusive jet, dijet and three-jet production in deep-inelastic scattering (DIS) [7,8] and used them to determine the strong coupling constant (at the mass $M_{Z}$ of the $Z$ boson) to be

$$
\alpha_{s}\left(M_{Z}\right)=0.1185 \pm 0.0016(\exp ) \pm 0.0040(\text { th }),
$$

taking into account absolute double-differential inclusive jet, dijet and three-jet cross section data as functions of $Q^{2}$ and the jet transverse momentum $p_{T}$. A more precise value was obtained from normalized jet cross sections, yielding

$$
\alpha_{s}\left(M_{Z}\right)=0.1165 \pm 0.0008(\exp ) \pm 0.0038(\text { th }) .
$$

Unsatisfactorily, only the value obtained by unnormalized results is in agreement with the current world average of

"michael.klasen@uni-muenster.de
$\alpha_{s}\left(M_{Z}\right)=0.1185 \pm 0.0006$ [9]. The latter uses only observables that are known to next-to-next-to-leading order (NNLO) of perturbative QCD, while the analysis of the H1 Collaboration was done in next-to-leading order (NLO) accuracy. The lack of knowledge of higherorder contributions becomes manifest in a bigger theoretical uncertainty due to scale variation in Eq. (1). The absolute double-differential cross section measurement of inclusive jets alone led $\mathrm{H} 1$ to a value of the strong coupling constant of

$$
\alpha_{s}\left(M_{Z}\right)=0.1174 \pm 0.0022(\exp ) \pm 0.0050(\text { th }) .
$$

In this paper, we compute the inclusive jet production DIS cross section for the first time including NNLO contributions, obtained from a unified threshold resummation formalism [10], and extract an approximate NNLO (aNNLO) value for the strong coupling constant. Our calculations are based on previous work on inclusive jet production in deep-inelastic scattering up to NLO [11]. They reduce, as we will see, the theoretical prediction and increase the central fit value of the strong coupling constant, improving the description of the final HERA data in particular at high photon virtuality $Q^{2}$.

\section{NNLO CONTRIBUTIONS TO JET PRODUCTION IN DIS}

The QCD factorization theorem allows to write the differential cross section for inclusive jet production in neutral-current DIS with high momentum transfer $Q^{2}=-q^{2}$ as a convolution of the partonic cross section $\mathrm{d} \sigma_{\gamma a}$ with the parton densities in the proton $f_{a / P}$ and the flux of photons in electrons $f_{\gamma / e}$ as 


$$
\begin{aligned}
\mathrm{d} \sigma= & \sum_{a} \int \mathrm{d} y f_{\gamma / e}(y) \\
& \times \int \mathrm{d} x_{P} f_{a / P}\left(x_{P}, \mu_{F}\right) \mathrm{d} \sigma_{\gamma a}\left(\alpha_{s}, \mu_{R}, \mu_{F}\right),
\end{aligned}
$$

where we define $y=(p \cdot q) /(p \cdot k)$ with $p$ and $k$ the momenta of the incoming proton and electron, respectively, and $q$ the momentum of the exchanged photon. In deepinelastic scattering the highly off-shell photon has no time to decay, so resolved photon contributions can safely be neglected.

From a unified threshold resummation formalism a master formula can be obtained that permits to compute soft and virtual corrections to arbitrary partonic hard scattering cross sections [10]. At NLO it reads

$$
\mathrm{d} \sigma_{a b}=\mathrm{d} \sigma_{a b}^{B} \frac{\alpha_{s}(\mu)}{\pi}\left[c_{3} D_{1}(z)+c_{2} D_{0}(z)+c_{1} \delta(1-z)\right],
$$

where for just one color-charged parton in the initial state we only need the formula for simple color flow. The functions

$$
D_{l}(z)=\left[\frac{\ln ^{l}(1-z)}{1-z}\right]_{+},
$$

with decreasing $l$ are the leading and subleading logarithms at partonic threshold $(z \rightarrow 1)$ in pair-invariant-mass kinematics, where $z=\left(p_{1}+p_{2}\right)^{2} /\left(q+p_{a}\right)^{2}$ and $p_{a}, p_{1}$ and $p_{2}$ are the momenta of the incoming and outgoing partons. The NNLO master formula is given in the reference cited above as Eq. (2.17), as are the general formulas for the coefficients $c_{i}$.

We state here the coefficients for the two partonic processes that contribute to jet production in DIS. For $\gamma^{*} q \rightarrow q g$, where $\gamma^{*}$ represents the off-shell photon, $g$ a gluon and $q$ a quark or an antiquark, we find

$$
\begin{gathered}
c_{3}=C_{F}-N_{C}, \\
c_{2}=2 C_{F} \ln \left(\frac{-u}{M^{2}}\right)+N_{C} \ln \left(\frac{t}{u}\right) \\
-C_{F} \ln \left(\frac{\mu_{F}^{2}}{M^{2}}\right)-\frac{3}{4} C_{F}-\frac{\beta_{0}}{4}
\end{gathered}
$$

and $c_{1}=c_{1}^{\mu}+T_{1}$ with

$$
c_{1}^{\mu}=-\frac{3}{4} C_{F} \ln \left(\frac{\mu_{F}^{2}}{M^{2}}\right)+\frac{\beta_{0}}{4} \ln \left(\frac{\mu_{R}^{2}}{M^{2}}\right) .
$$

For the second process $\gamma^{*} g \rightarrow q \bar{q}$ we find

$$
c_{3}=2\left(N_{C}-C_{F}\right) \text {, }
$$

$$
c_{2}=N_{C} \ln \left(\frac{t u}{M^{4}}\right)-N_{C} \ln \left(\frac{\mu_{F}^{2}}{M^{2}}\right)-\frac{3}{2} C_{F},
$$

and

$$
c_{1}^{\mu}=\frac{\beta_{0}}{4}\left[\ln \left(\frac{\mu_{R}^{2}}{M^{2}}\right)-\ln \left(\frac{\mu_{F}^{2}}{M^{2}}\right)\right] .
$$

These coefficients agree with those found in photoproduction for massless jets for the direct part [6]. For massive jets, additional logarithms depending on the jet radius $R$ appear (e.g. in $c_{2}$ ) [12], which are however irrelevant in the case $R=1$ as in the $\mathrm{H} 1$ analysis considered here [7]. Our calculation is analogous to the case of single-jet production in hadron-hadron collisions [13]. The above coefficients further depend on the QCD color factors $C_{F}=4 / 3$ and $N_{C}=3$, on the one-loop $\beta$-function $\beta_{0}=\left(11 N_{C}-2 n_{f}\right) / 3$ with $n_{f}$ the number of active quark flavors, the Mandelstam variables $t=\left(q-p_{1}\right)^{2}$ and $u=\left(p_{a}-p_{1}\right)^{2}$, the renormalization and factorization scales $\mu_{R}$ and $\mu_{F}$ and the fixed large invariant scale $M^{2}$, that in DIS is equal to $Q^{2}$, whereas in photoproduction it was the Mandelstam variable $s=\left(q+p_{a}\right)^{2}$. The part $T_{1}$ of $c_{1}$ does not contain any dependence either on the renormalization or the factorization scale. It includes the NLO virtual corrections and is not predicted by the threshold resummation formalism. If available, it can be read off from a full NLO calculation. For the case of transverse photon polarization it can be found in Eqs. (33), (34) and Appendix B of Ref. [11]. For longitudinal polarization we took the formula directly from the source code of the corresponding program Jetvip $[14,15]$, which calculates inclusive jet production in DIS to NLO accuracy. The two-loop quantities $\Gamma_{S}^{\prime(2)}, B_{j}^{\prime(2)}, \nu_{f_{i}, j}^{(2)}$ and $R$ appearing in the NNLO master formula were not given explicitly in [10] and could not be found in the respective literature. As in our previous work on jet photoproduction [6], they were neglected due to the fact that they are both subleading and not predicted by the threshold resummation formalism. In particular, for the few cases where it is known explicitly, the contribution of $\Gamma_{S}^{\prime(2)}$ has been shown to be small [16].

\section{COMPARISONS TO H1 DATA}

The NNLO contributions have been implemented in the code Jetvip for inclusive jet and dijet production in DIS, where the convolution over $z$ was already included for NLO initial-state corrections on the proton side. At NLO, we use of course our complete calculation and not only the logarithmically enhanced terms described above. As a numerical check, we have repeated the NLO analysis of inclusive single-differential jet production of the $\mathrm{H} 1$ Collaboration, performed with NLOJet $++[17]$ and presented in Refs. [7,8], and found excellent agreement, 
confirming previous successful comparisons of different NLO programs for jet production in DIS [14,17].

The measurement took place during the HERA-II running period with an integrated luminosity of $351 \mathrm{pb}^{-1}$. The beam energies were $27.6 \mathrm{GeV}$ for electrons or positrons and $920 \mathrm{GeV}$ for protons, which gives a center-of-mass energy of $319 \mathrm{GeV}$. The leptonic phase space was given by $150 \mathrm{GeV}^{2}<Q^{2}<15000 \mathrm{GeV}^{2}$ and $0.2<y<0.7$. The jet phase space was restricted to the rapidity interval $-1.0<\eta_{l a b}<2.5$, where $\eta_{l a b}$ is the pseudorapidity of a jet in the HERA lab frame. The cross section was measured differentially in the jet transverse momentum $p_{T}$ and the virtuality $Q^{2}$. Jets were reconstructed using the $k_{T^{-}}$ clustering algorithm [18] in the Breit frame, where exclusively electroweak processes can be ruled out by demanding a minimum of jet transverse momentum (here $p_{T}>7 \mathrm{GeV}$ ). In inclusive jet production in DIS, an almost identical fit result for $\alpha_{s}\left(M_{Z}\right)$ was obtained with the anti- $k_{T}$ algorithm. The jet radius was $R=1$. The perturbative scales were chosen to be

$$
\mu_{R}^{2}=\left(Q^{2}+p_{T}^{2}\right) / 2 \text { and } \mu_{F}^{2}=Q^{2} .
$$

The perturbative hard-scattering functions were convoluted with the MSTW2008 set of parton distribution functions in the proton with different fixed $\alpha_{s}\left(M_{Z}\right)$ values [19]. This PDF set especially offers the possibility to determine a bestfit $\alpha_{s}\left(M_{Z}\right)$. The number of active flavors was $n_{f}=5$, since sea contributions of a heavy top quark inside the proton can safely be neglected. Following the H1 analysis, we choose the PDF member with $\alpha_{s}\left(M_{Z}\right)=0.118$ in all plots shown in this paper, which together with the scale choice from Eq. (13) defines our central fit.

In Fig. 1, we compare our NLO (green dashed lines) and aNNLO (blue full lines) results to the experimental data of the H1 Collaboration (red points). The uncertainty bands are obtained by varying both scales simultaneously about the central scales up and down by a factor of two as it was also done by the $\mathrm{H} 1$ Collaboration. We have verified that our NLO results and uncertainty bands agree very well with those of the $\mathrm{H} 1$ analysis. Comparing our calculations to the measurements, we see that the data points lie in the error bands of both the NLO and the aNNLO calculations. For the $Q^{2}$ distribution (top), the scale uncertainty is of similar size at NLO and aNNLO at low $Q^{2}$ and is considerably reduced in aNNLO at higher $Q^{2}$ as expected. We checked that this tendency continues and becomes more pronounced for even higher $Q^{2}$, where unluckily we have no data to compare with. For the $p_{T}$ distribution (bottom), the scale uncertainty is not reduced from NLO to aNNLO, since analytically no logarithms of ratios of $\mu_{R}$ or $\mu_{F}$ over $p_{T}$ appear (see above). While in our previous analysis of jet photoproduction the jet transverse momentum $p_{T}$ ranged from 17 to $71 \mathrm{GeV}$ [6], its range is restricted in DIS and this analysis to lower values of 7 to $50 \mathrm{GeV}$, while the photon
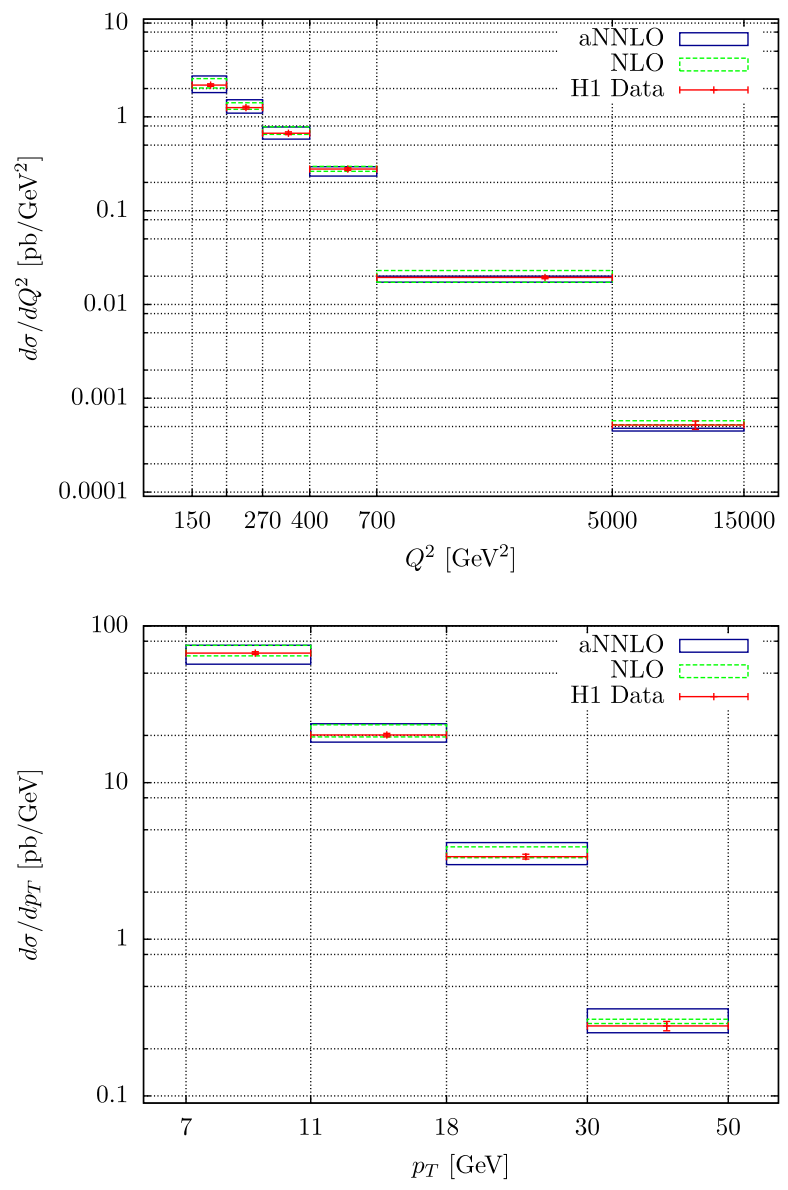

FIG. 1 (color online). Single-differential inclusive jet cross sections as a function of photon virtuality $Q^{2}$ (top) and jet transverse momentum $p_{T}$ (bottom) in NLO (green dashed lines) and aNNLO (blue full lines) with the corresponding scale uncertainty bands, obtained by varying $\mu_{R}$ and $\mu_{F}$ simultaneously by a factor of two up and down, compared to the final $\mathrm{H} 1$ data (red points, color online).

virtuality reaches values up to $(122.5 \mathrm{GeV})^{2}$. As a cross check of our calculation, we compare in Fig. 2 the approximate NLO (aNLO) with the exact NLO and the final $\mathrm{H} 1$ data for the $Q^{2}$ distribution. As one can see, the aNLO and NLO predictions agree very well within the respective scale uncertainties.

In Fig. 3, we show the same comparison as in Fig. 1 normalized to the central NLO result. We also depict here the central aNNLO result, which is always smaller than the NLO central result by approximately $6 \%$. The central $Q^{2}$ distribution and even more the central $p_{T}$ distribution agree better with the $\mathrm{H} 1$ data at aNNLO than at NLO, as they then lie right within the experimental uncertainties. While the central NLO results overestimate the measured cross sections, indicating that the value of the strong coupling constant $\alpha_{s}\left(M_{Z}\right)$ used at this order is too large, the central aNNLO results underestimate the data and require a slightly larger value of $\alpha_{s}$. The data are thus clearly sensitive to the strong coupling constant and can be used 


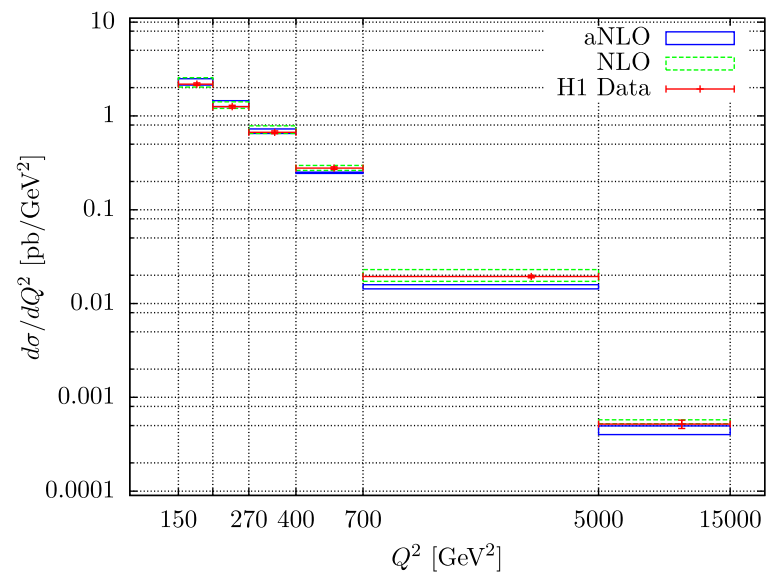

FIG. 2 (color online). Same as Fig. 1 (top), but comparing approximate (blue full lines) and exact NLO (green dashed lines) results with the corresponding scale uncertainty bands with each other and the final H1 data (red points, color online).

for an extraction not only at NLO, but also at aNNLO. However, we do not expect a significant reduction of the theoretical error from the scale uncertainty, in particular from the $p_{T}$ distribution.
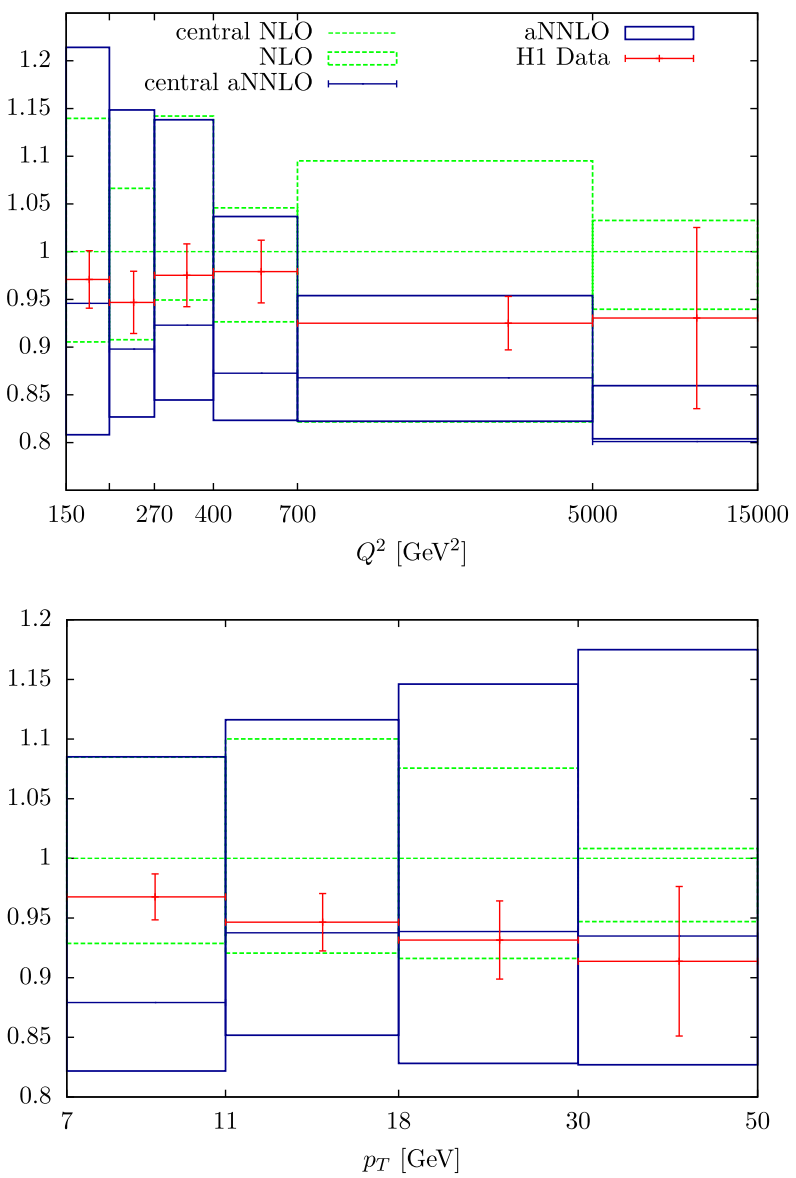

FIG. 3 (color online). Same as Fig. 1, but normalized to the central NLO predictions.

\section{DETERMINATION OF $\alpha_{s}$}

To determine the strong coupling constant from these comparisons, the theoretical calculations have to be performed with a set of parton densities in the proton obtained from global fits assuming different values of $\alpha_{s}\left(M_{Z}\right)$. For our analysis at aNNLO, we employ the latest fits of the MSTW group, which have been obtained with NNLO running of the coupling, evolution of the parton densities, deep-inelastic scattering and vector-boson production matrix elements [19]. Twenty-two different MSTW2008 NNLO members were used, which correspond to values of $\alpha_{s}\left(M_{Z}\right)=0.107$ to 0.127 . To compare the aNNLO best-fit $\alpha_{s}$ to a corresponding NLO $\alpha_{s}$ based on our full NLO calculation, we carry out the same approach with the NLO MSTW2008 parton distribution functions. These are available for the range of $\alpha_{s}\left(M_{Z}\right)=0.110$ to 0.130 .

The strong coupling constant $\alpha_{s}$ was determined by comparing the theoretical predictions at NLO and aNNLO to the experimental measurements by $\mathrm{H} 1$ and then finding the minimum value of the reduced $\chi^{2}$. We present here our results for the single-differential $p_{T}$ distribution, but have verified that fitting the $Q^{2}$ distribution yields very similar results. At NLO we find

$$
\alpha_{s}^{\mathrm{NLO}}\left(M_{Z}\right)=0.115 \pm 0.002(\exp ) \pm 0.005(\text { th }),
$$

where the central value is slightly lower than the one obtained by $\mathrm{H} 1$ from the unnormalized double-differential inclusive jet cross section [cf. Eq. (3)], but where the total experimental error and the theoretical error obtained from a simultaneous variation of the renormalization and factorization scales agree very well. At aNNLO, $\alpha_{s}$ gets shifted upwards, since we demonstrated above that the aNNLO contributions reduce the differential cross sections compared to NLO. We find

$$
\alpha_{s}^{\mathrm{aNNLO}}\left(M_{Z}\right)=0.122 \pm 0.002(\exp ) \pm 0.013(\text { th }),
$$

where the central value is now slightly above the world average, the experimental error is of course unchanged, and the theoretical error is slightly larger, reflecting the observation made above that the aNNLO calculation is not yet sufficiently stabilized by threshold logarithms at these values of $p_{T}$ and $Q^{2}$. The numerical situation would only improve at higher values of $Q^{2}$, where unfortunately no experimental data are available.

\section{CONCLUSIONS}

In conclusion, we have presented here a first calculation of inclusive-jet production in neutral-current deep-inelastic scattering up to NNLO of perturbative QCD. Leading and subleading logarithmic contributions were extracted from a unified threshold resummation formalism for virtual photon-parton scattering processes and shown to agree with 
those appearing in our full NLO calculations. The aNNLO contributions implemented in our NLO program improve the description of the final $\mathrm{H} 1$ data on inclusive-jet production in the $Q^{2}$ distribution and even more in the $p_{T}$ distribution, when the world average value of $\alpha_{s}$ is used and for central scale choices. The scale uncertainties are reduced only at the highest values of $Q^{2}$, where threshold corrections are most important. An aNNLO fit of these data with the MSTW2008 set of parton densities resulted in a new determination of the strong coupling constant at the mass of the $Z$ boson that increased the central fit value from below to above the current world average, but did not reduce the theoretical error.

\section{ACKNOWLEDGMENTS}

We thank W. Vogelsang for useful discussions. This work has been supported by the BMBF TheorieVerbund "Begleitende theoretische Untersuchungen zu den Experimenten an den Großgeräten der Teilchenphysik."
[1] C. Adloff et al. (H1 Collaboration), Eur. Phys. J. C 21, 33 (2001).

[2] S. Chekanov et al. (ZEUS Collaboration), Eur. Phys. J. C 21, 443 (2001).

[3] F. D. Aaron et al. (H1 and ZEUS Collaboration), J. High Energy Phys. 01 (2010) 109.

[4] M. Klasen, G. Kramer, and S. G. Salesch, Z. Phys. C 68, 113 (1995); M. Klasen and G. Kramer, Z. Phys. C 72, 107 (1996); 76, 67 (1997); M. Klasen, T. Kleinwort, and G. Kramer, Eur. Phys. J. direct C 1, 1 (2000); M. Klasen, G. Kramer, and B. Pötter, Eur. Phys. J. C 1, 261 (1998); M. Klasen and G. Kramer, Phys. Rev. D 56, 2702 (1997); Eur. Phys. J. C 71, 1774 (2011); M. Klasen, Rev. Mod. Phys. 74, 1221 (2002).

[5] S. Albino, M. Klasen, and S. Söldner-Rembold, Phys. Rev. Lett. 89, 122004 (2002).

[6] M. Klasen, G. Kramer, and M. Michael, Phys. Rev. D 89, 074032 (2014).

[7] V. Andreev et al. (H1 Collaboration), Eur. Phys. J. C 75, 65 (2015).

[8] D. A. Britzger (H1 Collaboration), Report No. DESY-THESIS-2013-045 (2013).
[9] K. A. Olive et al. (Particle Data Group Collaboration), Chin. Phys. C 38, 090001 (2014).

[10] N. Kidonakis, Int. J. Mod. Phys. A 19, 1793 (2004).

[11] B. Pötter, Eur. Phys. J. direct C 1, 1 (1999).

[12] D. de Florian and W. Vogelsang, Phys. Rev. D 76, 074031 (2007); M. C. Kumar and S. O. Moch, Phys. Lett. B 730, 122 (2014).

[13] N. Kidonakis and J. F. Owens, Phys. Rev. D 63, 054019 (2001).

[14] B. Pötter, Comput. Phys. Commun. 133, 105 (2000).

[15] M. Klasen, G. Kramer, and B. Pötter, Eur. Phys. J. C 1, 261 (1998).

[16] N. Kidonakis, Phys. Rev. D 73, 034001 (2006).

[17] Z. Nagy and Z. Trocsanyi, Phys. Rev. Lett. 87, 082001 (2001).

[18] S. Catani, Y.L. Dokshitzer, M. H. Seymour, and B. R. Webber, Nucl. Phys. B406, 187 (1993); S. D. Ellis and D. E. Soper, Phys. Rev. D 48, 3160 (1993).

[19] A. Martin, W. Stirling, R. Thorne, and G. Watt, Eur. Phys. J. C 64, 653 (2009). 